\title{
COVID-19, Ebola virus disease, and Nipah virus infection reclassification as novel acute immune dysrhythmia syndrome (n-AIDS): potential crucial role for immunomodulators
}

\author{
Mina T. Kelleni ${ }^{1}$ (D)
}

Received: 18 April 2021 / Accepted: 23 July 2021 / Published online: 6 August 2021

(c) The Author(s), under exclusive licence to Springer Science+Business Media, LLC, part of Springer Nature 2021

\begin{abstract}
In this manuscript, COVID-19, Ebola virus disease, Nipah virus infection, SARS, and MERS are suggested to be considered for a novel immunological reclassification as acute onset immune dysrhythmia syndrome (n-AIDS) due to altered monocytic, Th1/Th2, as well as cytokines and chemokines balances. n-AIDs is postulated to be the cause of the acute respiratory distress and multi-inflammatory syndromes which are described with fatal COVID-19, and immunomodulators are suggested to effectively manage the mentioned diseases as well as for other disorders caused by Th1/Th2 imbalance. Meanwhile, para COVID syndrome is suggested to describe various immune-related complications, whether before or after recovery, and to embrace a potential of a latent infection, that might be discovered later, as occurred with Ebola virus disease. Finally, our hypothesis has evolved out of our real-life practice that uses immunomodulatory drugs to manage COVID-19 safely and effectively.
\end{abstract}

Keywords COVID-19 $\cdot$ Ebola virus disease $\cdot$ Nipah virus infection $\cdot$ n-AIDS $\cdot$ Para COVID-19 syndrome

Almost three decades ago, a brilliant viewpoint has suggested that a dysregulated immunological switch in favor of Th2 type responses over the Th1 type response is associated with progression of HIV to AIDS and this switch was characterized by loss of the protective antiviral IL-2- and IFN- $\gamma$ production; furthermore, those expert researchers have suggested that thousands of seronegative, HIV-exposed (many on multiple occasions) individuals have generated strong Th1 dependent IL-2 responses to HIV antigens [1].

\section{Highlights}

- COVID-19 and selected other fatal viral diseases were shown to disrupt the immune system.

- Monocytic dysrhythmia and altered Th1/Th2 balance trigger COVID-19 mortality.

- n-AIDS is manifested by lymphopenia, causing ARDS and multi-inflammatory syndrome.

- Para COVID-19 syndrome describes immune-related complications whether manifest or latent.

- Immunomodulators might prove an important tool to combat COVID-19 and other fatal diseases.

\section{Mina T. Kelleni}

drthabetpharm@yahoo.com; mina.kelleni@mu.edu.eg

1 Pharmacology Department, College of Medicine, Minia University, Minya, Egypt
Interestingly, a similar imbalance in Th1/Th2 types with a Th2 favorable switch or failure in the activation of Th1 and reduced IFN- $\gamma$ production was observed in deceased SARS patients or critically ill MERS patients, whereas a Th1 strong response, which is pivotal in mediating virus-specific adaptive immunity, was observed in mild patients [2].

Importantly, CD4 + and CD8 + T cell cytokines were significantly diminished in COVID-19 patients as compared to healthy controls, and subverted $\mathrm{T}$ cell composition and/ or homeostasis were also suggested to share in COVID-19 pathogenesis, and IL-6 was implied to induce this immunopathological process in patients suffering from severe or critical COVID-19 [2, 3]. As an explanation, SARS CoV-2 was suggested to dysregulate the antiviral immune response at an early stage leading to number depletion and functional exhaustion of NK and CD8 $+\mathrm{T}$ cells which were restored in those who survived, and a recommendation to improve the immune response at the early stage of SARS CoV-2 infection was concluded [4].

However, though a variable immunological response was suggested to predict mortality in COVID-19 patients and Th2, Th17 cell, and Treg percentages were significantly lower in deceased COVID-19 cases than recovered and healthy control [5], yet COVID-19's high morbidity and mortality were still suggested to be related to low Th1 
immunity [6], and spike-specific Th1 cells capable of IL7dependent homeostatic proliferation were shown to predict survival from severe COVID-19 [7]. Similarly, un-sustained effective Th1 response and dominant Th2 response were demonstrated, in a prospective cohort of patients, to be related to a worse COVID-19 prognosis [8], and we suggest that their observed higher levels of IFN- $\gamma$ related to mortality might be, similar to other types of interferons, induced in critically ill patients in a final futile attempt to tune the untuned antiviral immunity [9] that exacerbate, instead of ameliorate, the induced immunopathic damage [10], and we would like to emphasize defective interferon response as a major culprit responsible for COVID-19 deterioration [11, 12].

In another explanation to the various clinical outcomes, ACE2 was suggested to regulate the immune response in SARS and SARS CoV-2 including activation of B cells, macrophages, Th1 cells and the inhibition of Treg cells and CD8 + T cells[13] and ACE2 [14] and other discovered [15] and potentially yet to be discovered genetic polymorphisms e.g. CARD 14 [16] might be reflected through different $T$ cell virus specific and other immunological responses. Thus, while some SARS CoV-2 exposed patients would remain symptoms-free, including asymptomatic seronegative COVID-19 patients [17], others suffer from mild-moderate or severe COVID-19. Similarly, some COVID-19 patients will recover smoothly, while others complain of COVID19 associated autoimmune complications [18] which are hypothesized to be due to transient immunosuppression of the innate and acquired immunity [19].

Thus, we have suggested a new terminology for SARS $\mathrm{CoV}-2$ induced dysregulated immune response, monocytic dysrhythmia [11], to be noted that an imbalanced immuneinflammatory response was previously described to drive the development of COVID-19 [20]. Meanwhile, we preferred the term dysrhythmia over dysregulation as we postulate that tuning the immune response deserves more research work that might lead to novel highly needed immunotherapeutic drugs.

Notably, we suggested to name para COVID syndrome [21] preferring it to post COVID to embrace a potential that SARS CoV-2 might induce immune-mediated disorders whether before or after recovery as well as it might persist latent, for yet unspecified period, in some cells and tissues [22] to induce, at least some of, what is currently being described of several post COVID19 diseases affecting the nervous system [23, 24]. Para COVID might also describe immunological reactivation of various types of herpes viruses which are described in critically ill COVID-19 patients [25], and we recommend further investigations to assess potential SARS CoV-2 direct latency or indirect persistent functional dysrhythmia, respectively, in some immune cells such as the migrating interstitial macrophages $[26,27]$. Obviously,
SARS CoV-2 is known to possess several adaptive and immune evasive differences from other coronaviruses, and our knowledge about RNA viruses and their capabilities to remain latent for long duration is still evolving; thus, SARS CoV-2 potential latency might eventually resemble the newly described latent Ebola virus (https://www.scien cemag.org/news/2021/03/new-ebola-outbreak-likely-spark ed-person-infected-5-years-ago).

In the same manner, a fourth RNA virus induced fatal disease; Ebola virus disease might also be considered to possess a similar $\mathrm{n}$-AIDS potential as regards to its induced dysregulation of the immune system [28], its long lasting T and $\mathrm{B}$ cell immunological dysfunction which was further described in Ebola survivors [29], as well as the dysregulated inflammatory and immunological immune response in both Ebola virus disease survivor and deceased cases [30]. Additionally, a fifth RNA virus with a high fatality rate; Nipah virus might be similarly reclassified as it has been shown to manipulate the inflammatory and immunological response including the interferon homeostasis [31]. Nipah virus infection was also shown to influence the pro-inflammatory and leucocyte attracting cytokines in a manner that determines the disease course [32] and to induce a dysregulated immune recruitment that led to acute vasculitis among other several induced immune dysregulatory mechanisms [33].

Taken together, we postulate that abnormal cytokine and chemokine, known and yet to be discovered, dependent lymphocyte distraction (clinically manifested by lymphopenia) into the lungs (causing ARDS) or away from the lungs to other organs (causing multiple inflammatory syndrome) might reason for COVID-19 pathogenesis and complications [34]. Moreover, we suggest that SARS, MERS, and SARS CoV-2, the three virulent RNA corona viruses which emerged in the past two decades, which are also anticipated to be joined by other potentially fatal similar viruses, together with Ebola virus disease and Nipah virus infection might be considered for a novel immunopathological reclassification, n-AIDS. We wish by introducing a novel academic classification (n-AIDS) and modifying an existing preliminary one (para COVID) to encourage academic and clinical researchers to evolve the topic further to be ready for the anticipated similar viruses/ pandemics in the coming years and to avoid any unnecessary delay that might cause terminology pollution. n-AIDS acknowledges the potential immune-linked pathogenesis of those viruses that might induce their complications and/ or fatalities as shown by their peculiar immune monocytic, Th1/Th2, and potentially other immune cells dysrhythmia that though seems hyperactive, it is practically deficient/ incompetent. We also encourage further investigations to assess potential SARS CoV-2 latency in some survivors, as recently shown for Ebola, to fully explore para COVID syndrome. 
Interestingly, though the three potentially fatal coronaviruses share some similarities with HIV [35], and that other potential similarities between SARS CoV-2 and Nipah virus have been also described [36], these viruses and Ebola virus also differ from HIV in several important aspects including their specific immunological targets and their main tendency for acute progressive onset and complications, and hence $\mathrm{n}$-AIDS is suggested to be more scientifically accurate to classify them.

Accordingly, we suggest that our suggested novel classification of acute immune-dysrhythmic syndrome might properly guide us in our quest for a potential cure; as it is only when we know the cause, we can insightfully figure out the therapy, and we recommend to focus on immune-modulation as a potential effective COVID-19 therapy [6, 11, 37], Ebola virus disease [38], and Nipah virus infection [31]. Moreover, we hypothesize that our evolved real-life immunomodulatory COVID-19 management protocol [11,37] that guided us, through its remarkable clinical efficacy against COVID-19, to this hypothesis might also prove beneficial when tested in clinical trials for early management of the mentioned and other RNA viruses that would be classified to cause n-AIDS as well as to potentially manage other diseases caused by altered Th1/ Th2 balance.

Finally, I agree with an expert who said: "I think there is just so much going on here, and we are just putting our foot the river. I'll just leave you with this thought: we are closer to the beginning than the end of this" (https://www.healio.com/ news/rheumatology/20210416/data-supports-covid19-as-autoi mmunity-trigger-in-patients-without-preexisting-imids).

Acknowledgements This work is dedicated to the soul of Dr. George Habib Bebawi (1938-2021), a great Egyptian American worldrenowned theologian, patristic scholar, and a former director of studies at the Institute for Christian Orthodox Studies, at Cambridge, England. He has bravely and relentlessly fought for what he truly believed orthodox while struggling against a lifelong oppression and injustice that has not ended until his departure. May his memory be everlasting. I wish also to express my deepest gratitude for Immunologic Research and its honorable Editor in Chief Professor Nicola Bizzaro for the very professional handling of this manuscript as highly constructive and very insightful remarks that came from honorable anonymous peer reviewers have significantly improved the quality of the represented data.

\section{Declarations}

Conflict of interest The author declares that there is no conflict of interest.

\section{References}

1. Clerici M, Shearer GM. A TH1 $\rightarrow \mathrm{TH} 2$ switch is a critical step in the etiology of HIV infection. Immunol Today. 1993;14(3):107-11.
2. Zhang Y-Y, et al. The comparative immunological characteristics of SARS-CoV, MERS-CoV, and SARS-CoV-2 coronavirus infections. Front Immunol. 2020. https://doi.org/10.3389/ fimmu.2020.02033.

3. Rupp J, et al. T cell phenotyping in individuals hospitalized with COVID-19. J Immunol. 2021;206:1478.

4. Zheng M, et al. Functional exhaustion of antiviral lymphocytes in COVID-19 patients. Cell Mol Immunol. 2020;17(5):533-5.

5. Sami R, et al. Characterizing the immune responses of those who survived or succumbed to COVID-19: can immunological signatures predict outcome? Cytokine. 2021;140:155439.

6. Gupta A. Is immuno-modulation the key to COVID-19 pandemic? Indian J Orthopaedics. 2020;54(3):394-7.

7. Neidleman J, et al. Distinctive features of SARS-CoV-2-specific T cells predict recovery from severe COVID-19. MedRxiv. 2021;587:270.

8. Gadotti AC, et al. IFN- $\gamma$ is an independent risk factor associated with mortality in patients with moderate and severe COVID-19 infection. Virus Res. 2020;289:198171-198171.

9. Galani I-E, et al. Untuned antiviral immunity in COVID-19 revealed by temporal type I/III interferon patterns and flu comparison. Nat Immunol. 2021;22(1):32-40.

10. Lopez L, et al. Dysregulated interferon response underlying severe COVID-19. Viruses. 2020;12(12):1433.

11. Kelleni MT. NSAIDs/nitazoxanide/azithromycin repurposed for COVID-19: potential mitigation of the cytokine storm interleukin-6 amplifier via immunomodulatory effects. Expert Rev Anti Infect Ther. 2021. https://doi.org/10.1080/14787210.2021. 1939683.

12. Bastard P, et al. Autoantibodies against type I IFNs in patients with life-threatening COVID-19. Science. 2020;370(6515):eabd4585.

13. Luo J, et al. The potential involvement of JAK-STAT signaling pathway in the COVID-19 infection assisted by ACE2. Gene. 2021;768:145325.

14. Kelleni M. ACE2 Polymorphisms reflected on the immune and apelinergic peptide systems: potential COVID-19 tools for risk stratification and therapy. Authorea (Preprint). 2021. https://doi. org/10.22541/au.162126670.06196092/v2.

15. Pairo-Castineira E, et al. Genetic mechanisms of critical illness in Covid-19. Nature. 2020;591:92-8.

16. Kelleni M. NSAIDs Immunomodulation in COVID-19 might inhibit SARS CoV-2 ORF proteins induced caspase activation, necroptosis and endoplasmic reticulum stress. Authorea (Preprint). 2021. https://doi.org/10.22541/au.162126663.38675715/ v2.

17. Lou B, et al. Serology characteristics of SARS-CoV-2 infection since exposure and post symptom onset. Eur Respir J. 2020;56:2000763.

18. Bhadelia N, et al. Distinct autoimmune antibody signatures between hospitalized acute COVID-19 patients, SARS-CoV-2 convalescent individuals, and unexposed pre-pandemic controls. MedRxiv. 2021. https://doi.org/10.1101/2021.01.21.21249176.

19. Cañas CA. The triggering of post-COVID-19 autoimmunity phenomena could be associated with both transient immunosuppression and an inappropriate form of immune reconstitution in susceptible individuals. Med Hypotheses. 2020;145:110345.

20. Blanco-Melo D, et al. Imbalanced host response to SARS-CoV-2 drives development of COVID-19. Cell. 2020;181(5):1036-45. e1039.

21. Kelleni M. SARS CoV-2 might exploit cells of the innate immune system to induce the novel acute immune dysrhythmic syndrome (n-AIDS) and para COVID-19 syndrome: a case report. Authorea (Preprint). 2021. https://doi.org/10.22541/au. $162126698.87303239 / \mathrm{v} 2$. 
22. Pietsch H, et al. Proof of SARS-CoV-2 genomes in endomyocardial biopsy with latency after acute infection. Int J Infect Dis. 2021;102:70-2.

23. Pezzini A, Padovani A. Lifting the mask on neurological manifestations of COVID-19. Nat Rev Neurol. 2020;16(11):636-44.

24. Taquet M, et al. 6-month neurological and psychiatric outcomes in survivors of COVID-19: a retrospective cohort study using electronic health records. Lancet Psychiatry. 2021;8:416-27.

25. Simonnet A, et al. High incidence of Epstein-Barr virus, cytomegalovirus, and human-herpes virus- 6 reactivations in critically ill patients with COVID-19. Infect Dis Now. 2021;51:296.

26. Tavazzi G, et al. Myocardial localization of coronavirus in COVID-19 cardiogenic shock. Eur J Heart Fail. 2020;22(5):911-5.

27. Liegeois $\mathbf{M}$, et al. The interstitial macrophage: a longneglected piece in the puzzle of lung immunity. Cell Immunol. 2018;330:91-6.

28. Falasca L, et al. Molecular mechanisms of Ebola virus pathogenesis: focus on cell death. Cell Death Differ. 2015;22(8):1250-9.

29. Wiedemann A, et al. Long-lasting severe immune dysfunction in Ebola virus disease survivors. Nat Commun. 2020;11(1):3730.

30. Colavita F, et al. Inflammatory and humoral immune response during Ebola virus infection in survivor and fatal cases occurred in Sierra Leone during the 2014-2016 outbreak in West Africa. Viruses. 2019;11(4):373.

31. Pelissier R, et al. Recent advances in the understanding of Nipah virus immunopathogenesis and anti-viral approaches. F1000Research. 2019. https://doi.org/10.12688/f1000research.19975.1.

32. Satterfield BA, et al. The immunomodulating V and $\mathrm{W}$ proteins of Nipah virus determine disease course. Nat Commun. 2015;6(1):7483.
33. Prescott J, et al. The immune response to Nipah virus infection. Adv Virol. 2012;157(9):1635-41.

34. Kelleni MT. Early use of non-steroidal anti-inflammatory drugs in COVID-19 might reverse pathogenesis, prevent complications and improve clinical outcomes. Biomed Pharmacother. 2021;133:110982.

35. Saleemi MA, et al. Emergence and molecular mechanisms of SARS-CoV-2 and HIV to target host cells and potential therapeutics. Infect Genet Evol J Mol Epidemiol Evol Genet Infect Dis. 2020;85:104583-104583.

36. Roe K. Explanation for COVID-19 infection neurological damage and reactivations. Transbound Emerg Dis. 2020;67(4):1414-5.

37. Kelleni M. NSAIDs/nitazoxanide/azithromycin immunomodulatory protocol used in adults, geriatric, pediatric, pregnant, and immunocompromised COVID-19 patients: a prospective observational study and case-series. Authorea (Preprint). 2021. https:// doi.org/10.22541/au.162126601.15715282/v5.

38. Bixler SL, et al. Discovering drugs for the treatment of Ebola virus. Curr Treat Options Infect Dis. 2017;9(3):299-317.

Publisher's Note Springer Nature remains neutral with regard to jurisdictional claims in published maps and institutional affiliations. 